\title{
Perforated gastric and duodenal ulcers: treatment options
}

\begin{abstract}
This paper focuses on a prospective nonrandomized review of patients undergoing surgery for perforated gastric and duodenal ulcers.

Patients and methods: A total of 198 patients with perforated gastric and duodenal ulcers were enrolled in the study between 2011 and 2016. The mean age of patients was 42 years. The disease was more common in men $(87.3 \%)$ than in women $(12.6 \%)$. The incidence of duodenal ulcer perforation was $86.3 \%$. Anti-helicobacter therapy was administered to $33.8 \%$ of patients before perforation. In $5.6 \%$ of cases, recurrent ulcer perforation was established. The majority of patients $(78.8 \%)$ were admitted within the first 12 hours, while $7.5 \%$ - 24 hours after perforation. The APACHE II scoring system was used to measure shock in $5.5 \%$ of cases. A score up to 6 was determined in $37.8 \%$ of patients, up to 12 - in $47.9 \%$, higher scores (more than 12) were measured in the remaining patients. Physical examination and diagnosis included clinical methods, abdominal X-ray, gastroduodenoscopy, ultrasound, laparoscopy, and the Boey risk scores.

Results: Diagnostic laparoscopy was performed in $79.3 \%$ of patients, the diagnosis of concealed perforation was confirmed by gastroduodenoscopy. Video-assisted laparoscopy was performed in $59 \%$ of patients, $14.7 \%$ - underwent combined minimally invasive surgery (abdominal laparascopic examination and surgical intervention in the stomach or duodenum using a minimally invasive technique) and $26.3 \%$ - via laparotomy. Laparoscopic surgery was possible on the basis of gastroduodenoscopy, laparoscopy (perforation diameter, a correlation between the size of ulcer and perforation diameter, periulcerous infiltration sizes). Postoperative complications were recorded in $8.1 \%$ of cases, the mortality rate was $4.5 \%$.
\end{abstract}

Keywords: perforated gastric and duodenal ulcer, diagnostics, surgical treatment
Volume 3 Issue I - 2018

ShamilV Timerbulatov, Vil M Timerbulatov, RI Khisamutdinova, MakhmudV Timerbulatov Department of Surgery, Bashkir State Medical University, Russia

Correspondence: ShamilV Timerbulatov, Department of Surgery, Bashkir State Medical University, Russia, Email timersh@yandex.ru

Received: January 31, 2018 | Published: February 26, 2018

\section{Introduction}

Perforated gastric and duodenal ulcers (PGDU) continue to be one of surgical problems accompanied by a $30 \%$ mortality rate and complications up to $50 \%$ including an acute abdominal syndrome, limited or generalized peritonitis and a high risk of sepsis. ${ }^{1}$ of the complications encountered, perforation ranks second in frequency after bleeding (the ratio is 1:6) and is the most common indication for emergency surgery for peptic ulcer. ${ }^{2-4}$ In 2006 , more than 150.000 patients with peptic ulcer (PU) complications were hospitalized in the USA alone. The total number of complications due to perforation $(\mathrm{n}=14500-9 \%)$ was as much as 7 times less than bleeding. Peptic ulcer perforations caused death in $37 \%$ of cases $^{2}$ and more than one in every admission related to peptic ulcer complicated by perforation was fatal. In ulcer perforation, the mortality rate was about 5 times higher than that in bleeding from the site of perforation. It was also the most significant death factor in US hospitals between 1993 and 2006 (OR: 12.1 (95\% CI: 9,8-149)). ${ }^{2}$

Over the past $30-40$ years, the European mortality rate associated with PGDU has been stable despite some progress made in perioperative management, visualization and surgical treatment techniques. $^{5-7}$
An imbalance between protective and ulcer-promoting factors in the pathogenesis of PU is evident. However, the causes of perforation in some patients need to be elucidated. Ulcerogenesis is associated with infections (H. pylori), disruptions in the mucosal protective barriers (the administration of certain drugs), and the excessive secretion of acid, although appropriate risk assessment and the impact of each factor are not sufficiently studied. ${ }^{8}$ Only about one third of patients with PGDU have a history of PU. In some patients, small perforations $(<5 \mathrm{~mm}$ in diameter) without large mucosal defects occur. This fact may suggest that the size of an ulcer is not related to perforation risk development, although in a number of patients with large defects (a few $\mathrm{cm}$ in diameter) perforations may occur. The latest development has been the recognition of the role of Helicobacter pylori in the pathogenesis of perforation..$^{9,10}$

A total of 198 patients with PGDU were treated at the Ufa Emergency hospital between 2011 and 2016. The mean age of patients was 42 years. The disease was more common among males - 173 $(87.3 \%)$ than females - 25 (12.6\%). Perforated duodenal ulcers were detected in 171 patients $(86.3 \%)$, gastric localization was found in 27 patients $(13.7 \%)$.

Perforated duodenal ulcers occurred most commonly among young patients, whereas gastric ulcers - in the aged. Of the total number, 8 
patients $(4 \%)$ were given nonsteroid anti-inflammatory drugs before perforation. Of interest, before admission related to PGDU anti-ulcer therapy was administered to 184 patients $(92,9 \%)$. Among them, 67 patients $(33.8 \%)$ received anti-helicobacter therapy. Out of them, only in one fifths of patients the treatment outcomes were assessed by fibrogastoduodenoscopic diagnostic testing for the presence of H.pylori. Eleven patients (5.6\%) underwent surgery for recurrent gastric ulcer perforations (4) and duodenal ulcer perforations (7). Perforation combined with other complications was encountered in 6 cases $(3 \%)$ including gastroduodenal hemorrhage in 1 patient $(0.5 \%)$, stenosis of the pyloris in 1 case $(0.5 \%)$, ulcer penetrated into the head of the pancreas and small omentum in 4 cases $(2 \%)$. The following distribution of admissions was established: $71,2 \%$ of patients were admitted within 6 hours after disease, $15(7.6 \%)$ - from six to twelve hours, $27(13.6 \%)$ - from 12 to 24 hours, $12(6 \%)$ - from 24 to 48 hours, and 3 patients $(1.5 \%)$ had very delayed presentation (over 48 h). Shock on admission was recorded in $5.5 \%$ patients.

The APACHE II scoring system was used to assess patients' illness severity: a score of up to 6 was in 75 patients $(37.8 \%)$, up to 12 - in $95(47.9 \%)$, from 12 to 18 - in $22(11.1 \%)$, and over 18 - in 6 patients $(2.8 \%)$.

In PGDU diagnostics, along with abdominal radiography fibrogastroduodenoscopy was used. When necessary, re-radiography following endoscopy, ultrasound of the abdomen and diagnostic laparoscopy could be applied. Laparoscopy was performed in $79.3 \%$ of patients.

We faced certain diagnostic difficulties in patients with concealed peptic ulcer ( $\mathrm{n}=11$ patients). In these cases the diagnosis was established ultrasonographically and gastroduodenoscopically. In this patient group, the time of final updated diagnostics did not exceed 3 hours. In the remaining patients the diagnosis was verified within the first two hours after admission.

Endoscopic diagnostics - gastroduodenoscopy is essential in determining the location and diameter of the perforation. The following criteria are the most applicable to choose the perforation suture:

\section{The size of ulcer. \\ 2. The perforation diameter. \\ 3. Periulcerous infiltration sizes. \\ 4. The peritonitis extent.}

We determine the location of the ulcer and its size, perforation size using gastroduodenoscopy. Occasionally there may be difficulty in determining the periulcerous infiltration size. Although laparoscopy is believed to be a more preferable technique, but with marked tissue infiltration it is hardly helpful for suturing large perforations. A laparotomic approach was applied with perforations over $10 \mathrm{~mm}$, generalized peritonitis, and the presence of expressed intra-abdominal hypertension over $25 \mathrm{~mm} \mathrm{Hg}$.

To choose proper surgery, we apply the following technique: during gastroduodenoscopy we use visualization assessment of the ulcer and perforation size and determine their correlation. Periulcerous infiltration markedness is designated as $\mathrm{Ku}$ coefficient for the ulcer diameter $(\mathrm{Du})$ to the perforation diameter $(\mathrm{Dp})$. The intraoperative analysis ( $\mathrm{n}=44$ patients) has shown that the Du:Dp correlation defines the wall thickness of ulcer infiltration and may determine the markedness of periulcerous infiltration $(\mathrm{Ku})$. With $\mathrm{Ku}$ coefficient less than 2 insignificant periulcerous infiltration or its absence is determined and laparoscopic suture of the gastric or duodenal ulcer perforation with a single-row suture is used. With $\mathrm{Ku}$ equal to 2 or over, with marked periulcerous infiltration a laparotomic approach is used for suturing the perforation in conjunction with the ulcer dissection.

\section{Discussion}

Despite the availability of long-term experience in treating patients with PGDU, some issues remain disputable. It is necessary to reveal correlating risk factors for improving potential treatment outcomes. Thus, the most widely used prediction model is the Boey clinical score based on the presence of serious diseases, preoperative shock and long standing perforation $\left(>24\right.$ h). ${ }^{11}$ However, its positive prognostic value in $94 \%$ of cases was not confirmed by subsequent studies. ${ }^{12-14}$

Other prognostic criteria specifically for PGDU were proposed. ${ }^{11}$ Nevertheless; none of them was confirmed in external cohorts. This prevents their generalization. In addition, some common surgical systems and scores were used.

The authors' technique based on the correlation between the size of the ulcer and perforation diameter simplifies the treatment option and reduces the number of complications. So, complications in the patient group operated on laparotomically were higher (OR: 0.240 (95\%), CI: 0.063-0.656) compared with laparoscopic interventions. With patient randomization, if the coefficient was less than 2 and without it (44 patients with the coefficient and 73 without it) complications were significantly lower ( 1 and 3 complications, respectively) (OR:0.543 (95\%) CI 0.055-5.385). Similar results were obtained in patients with an open procedure (OR:0.210 (95\%) CI 0.042-1.042): in the presence of the coefficient, there were 2 complications in 38 patients, whereas without it - 9 complications in 43 patients.

All 198 patients with PGDU underwent surgery predominantly within the first two hours after admission. Patients with marked intoxication, unstable hemodynamics, signs of peritonitis, infectious and toxic shock ( $\mathrm{n}=22$ patients) needed short-term preoperative management. The indications for management of these patients included central venous pressure up to $8-12 \mathrm{~mm} \mathrm{Hg}$, mean arterial pressure $>65 \mathrm{~mm} \mathrm{Hg}, \mathrm{SvO}_{2}>70 \%$, diuresis $>0.5 \mathrm{ml} /\left(\mathrm{kg}^{*} \mathrm{~h}\right)$.

Patients with PGDU were treated using three surgical interventions: a video-assisted laparoscopic approach $(\mathrm{n}=117(59 \%))$; combined minimally invasive approaches - abdominal laparoscopic examination and interventions in the stomach or duodenum using a mini-laparotomic approach $(\mathrm{n}=29(14.7 \%))$ and surgery using a routine laparotomic approach $(n=52(26.3 \%))$.

The indications for laparatomy were primarily established in 8 patients with generalized peritonitis, a critical state according to the APACHE II scores (> 18 and over), delayed presentation of patients ( $>24 \mathrm{~h}$ from onset).

According to the literature data, the frequency of conversion to laparotomy is $28.5 \%$, average is about $12.4 \%$. Conversion is recommended in the presence of technical difficulties related to suture 
of the perforation site, when the ulcer location is not demonstrable and there are large perforations $(>10 \mathrm{~mm}){ }^{15,16}$ The use of omenoplasty may reduce the possibility of conversion and with perforations over $10 \mathrm{~mm}$.

At surgery, concealed perforation was revealed in 11 patients (5.5\%), localized peritonitis - in $29(4.6 \%)$, diffuse - in $32(16.1 \%)$ and diffuse peritonitis in 126 patients $(63.6 \%)$. According to clinical criteria, the reactive phase of peritonitis was established in 47 patients $(23.7 \%)$, toxic - in $142(71.7 \%)$ and terminal - in 9 patients $(4.5 \%)$. Laparoscopic approaches have a number of advantages to most patients with PGDU. On these bases they were performed in 146 patients $(73.6 \%)$ including combined ones with ulcer suturing using a mini-laparotomic approach in 29 patients (14.6\%).

Contraindications to laparoscopy in PGDU were considered to be the patient's critical health state (the total APACHE scores >18), terminal peritonitis phase, diffuse peritonitis with multiple abdominal abscesses, the necessity in intubation and decompression of the bowel, generalized peritonitis along with surgery for the abdominal cavity organs. In the authors' view, additional objective assessment criteria for laparoscopy are the grade of intra-abdominal hypertension syndrome and the level of abdominal perfusion pressure (determined as a difference between average arterial and intra-abdominal pressure). Critical criteria are an increase in intra-abdominal pressure $>20 \mathrm{~mm}$ $\mathrm{Hg}$ and abdominal perfusion pressure $<65 \mathrm{~mm} \mathrm{Hg} .{ }^{6}$ The most safe is laparoscopy with the Boey risk score of $0-1{ }^{11}$

At combined minimally invasive surgery, laparoscopic examination of the abdomen was initially performed, and then suture of the perforation site was done using a mini-laparotomic approach. This type of surgery was applied when there were technical difficulties with laparoscopic suture, the large size of perforation, marked infiltration changes around the perforation site, organ abnormalities, when it was impossible to suture the perforation and in the presence of combined complications (perforation + bleeding or + stenosis, etc.).

Grade I perforated ulcer (the perforation diameter $<2 \mathrm{~mm}$ ) was seen in 4 patients $(2 \%)$, Grade II (diameter $2-5 \mathrm{~mm})$ - in 96 patients $(48.5 \%)$, Grade III (diameter 6-10 mm) - in 75 patients $(37.8 \%)$ and Grade IV (diameter $>10 \mathrm{~mm}$ ) - in 22 patients $(11,1 \%)$.

In our clinic, suture of the perforation site was performed in 191 patients $(96.5 \%)$, suture in conjunction with proximal selective vagotomy - in $5(2 . \%)$ and gastric resection - in 2 patients (1\%). To suture, one-row vicril sutures $(\mathrm{n}=88-46 \%)$, two-row $(\mathrm{n}=71-38.7 \%)$, sometimes suture with two-row sutures with ulcer resection $(\mathrm{n}=18-$ $9.4 \%)$ and two-row sutures with omenoplasty $(\mathrm{n}=11-5.7 \%)$ were used.

One-row sutures were used for small perforation sites (grades I-II) and in the absence of marked inflammatory changes around the ulcer. Two-row sutures were used for perforation sites of grades III-IV and in the presence of inflammatory changes around the ulcer. Two-row sutures with omenoplasty were used for perforation sites of grades III-IV with marked changes (inflammatory wall $>10 \mathrm{~mm}$ ) as a rule applying laparotomic or mini-laparotomic approaches.

Suture with ulcer defect resection was used with pyloroduodenal localization due to the eruption of sutures, stenosis of the lumen of the pylorus or the duodenum, as well as when pyloplasty was necessary.
Mandatory gastric resections were performed in one case when malignancy of the gastric localization was suspected. The second case was associated with the presence of a giant ulcer and impossibility to suture the perforation site.

Postoperative complications developed in 16 patients $(8.1 \%)$ including failure of sutures in one case $(0.5 \%)$, intra-abdominal abscesses - $6(3.1 \%)$, suppuration of operation wounds in 9 cases (4.5\%). After laparoscopic suture and examination, the suture failure was seen in 2 cases, after laparotomy - in 4 cases (OR:0.104 (95\%) CI 1.063-1.130). Suppuration of wounds occurred after an open procedure (8), in one case - after mimi-laparotomy

Postoperative lethality in the study group was $4.5 \%$ (9 patients). The causes for unfavorable outcomes were septic shock, uncured diffuse peritonitis, multi-organ insufficiency, thromboembolia of the lung artery. All those patients who died had undergone surgery using a classical laparotomic approach in the state of shock due to diffuse peritonitis with intra-abdominal abscesses. All of them were admitted after 24 hours after onset, and 3 patients after 48 hours.

\section{Conclusion}

Perforated gastric and duodenal ulcers remain a challenge in modern surgery due to high rates of complications and mortality. At the present time the issues of the pathogenesis of gastroduodenal ulcer perforation, mechanisms of regeneration as well as the risk of sepsis need to be better considered. In cases when the perforation is large $(>20 \mathrm{~mm})$ or inflamed tissues are friable there is difficulty in achieving safe healing of the wound by a primary suture. If the first attempt of the defect closure fails, the next one may also result in failure. In such cases resection should be considered, taking into account that large gastric ulcers or recurrent perforations must serve as an important indicator of malignancy which occurs in about $30 \%$ of patients. ${ }^{16,17}$ In these circumstances, gastric resection, partial resection of the stomach with gastrounostomy or insertion of a T-like drainage should be conducted if the defect is located in the duodenum. ${ }^{18}$

In recent years, to treat PGDU the endoscopic application of clips or stenting, ${ }^{19,20}$ the use of biodegradable material to close the defect in the ulcer site,,$^{21}$ mesenchymal stem cells to improve healing have been proposed..$^{22-27}$

\section{Acknowledgements}

None.

\section{Conflicts of interest}

The authors declare there is no conflict of interest.

\section{References}

1. Møller MH, Adamsen S, Thomsen RW, et al. Multicentre trial of a perioperative protocol to reduce mortality in patients with peptic ulcer perforation. Br J Surg. 2011;98(6):802-810.

2. Wang YR, Richter JE, Dempsey DT. Trends and outcomes of hospitalizations for peptic ulcer disease in the United States, 1993 to 2006. Ann Surg. 2010;251(1):51-58.

3. Lau JY, Sung J, Hill C. Systematic review of the epidemiology of complicated peptic ulcer disease: incidence, recurrence, risk factors and mortality. Digestion. 2011;84(2):102-113. 
4. Güzel H, Kahramanca S, Şeker D. Peptic ulcer complications requiring surgery: What has changed in the last 50 years in Turkey. Turk $J$ Gastroenterol. 2014;25(2):152-155.

5. Sonnenberg A. Time trends of ulcer mortality in Europe. Gastroenterology. 2007;132(7):2320-2327.

6. Lanas A, García RLA, Polo TM. et al. The changing face of hospitalisation due to gastrointestinal bleeding and perforation. Aliment Pharmacol Ther. 2011;33(5):585-591.

7. Thorsen K, Glomsaker TB, Meer A, et al. Trends in diagnosis and surgical management of patients with perforated peptic ulcer. J Gastrointest Surg. 2011;15(8):1329-1335

8. Horowitz J, Kukora JS, Ritchie WP. All perforated ulcers are not alike. Ann Surg. 1989;209(6):693-696.

9. Salama NR, Hartung ML, Müller A. Life in the human stomach: persistence strategies of the bacterial pathogen Helicobacter pylori. Nat Rev Microbiol. 2013;11(6):385-399.

10. Cid TP, Fernández MC, Benito MS. et al. Pathogenesis of Helicobacter pylori infection. Helicobacter. 2013;18(1):12-17.

11. Boey J, Choi SK, Poon A. Risk stratification in perforated duodenal ulcers. A prospective validation of predictive factors.Ann Surg. 1987; 205(1):22-26

12. Thorsen K, Søreide JA, Søreide K. Scoring systems for outcome prediction in patients with perforated peptic ulcer. Scand J Trauma Resusc.Emerg Med. 2013;21:25.

13. Thorsen K, Søreide JA, Søreide K. What is the best predictor of mortality in perforated peptic ulcer disease? A population-based, multivariable regression analysis including three clinical scoring systems. $J$ Gastrointest Surg. 2014;18(7):1261-1268.

14. Buck DL, VesterAM, Møller MH. Accuracy of clinical prediction rules in peptic ulcer perforation: an observational study. Scand J Gastroenterol. 2012;47(1):28-35.

15. Søreide K, Thorsen K, Søreide JA. Strategies to improve the outcome of emergency surgery for perforated peptic ulcer. Br J Surg. 2014;101(1): e51-64.
16. Ergul E, Gozetlik EO. Emergency spontaneous gastric perforations: ulcus versus cancer. LangenbecksArch. Surg. 2009;394(4): 643-646.

17. Kumar P, Khan HM, Hasanrabba S. Treatment of perforated giant gastric ulcer in an emergency setting. World J Gastrointest Surg. 2014;6(1):5-8.

18. Gupta V, Singh SP, Pandey A, et al. Study on the use of T-tube for patients with persistent duodenal fistula: is it useful?. World J Surg. 2013;37(11):2542-2545.

19. Parodi A, Repici A, Pedroni A, et al. Endoscopic management of GI perforations with a new over-the-scope clip device (with videos). Gastrointest Endosc. 2010;72(4):881-886.

20. Mönkemüller K, Peter S, Toshniwal J, et al. Multipurpose use of the 'bear claw' (over-the-scope-clip system) to treat endoluminal gastrointestinal disorders. Dig Endosc. 2014;26(3):350-357.

21. Bertleff MJ, Stegmann T, Liem RS, et al. Comparison of closure of gastric perforation ulcers with biodegradable lactide-glycolide-caprolactone or omental patches. JSLS. 2009;13(4):550-554.

22. Sanabria AE, Morales $\mathrm{CH}$, Villegas MI. Laparoscopic repair for perforated peptic ulcer disease. Cochrane Data base Syst Rev. 2004. 19;(4):CD004778.

23. Surapaneni S, Reddy AVB. The perforation-operation time interval; an important mortality indicator in peptic ulcer perforation. J Clin Diagn Res. 2013;7(5):880-882.

24. Wilhelmsen M, Møller MH, Rosenstock S. Surgical complications after open and laparoscopic surgery for perforated peptic ulcer in a nationwide cohort. Br J Surg. 2015;102(4):382-387.

25. Bertleff MJ, Lange JF. Laparoscopic correction of perforated peptic ulcer: first choice? A review of literature. Surg Endosc. 2010;24(6):12311239.

26. Lobankov VM. Problems of surgical treatment of peptic ulcer. Public health. 2005;7-C:25-29.

27. Buck DL1, Vester AM, Møller MH, et al. Surgical delay is a critical determinant of survival in perforated peptic ulcer. $\mathrm{Br} J$ Surg. 2013;100(8):1045-1049. 